\title{
Bounding the finite-size error of quantum many-body dynamics simulations
}

\author{
Zhiyuan Wang $\odot,{ }^{1,2, *}$ Michael Foss-Feig, ${ }^{3}$ and Kaden R. A. Hazzard ${ }^{1,2}$ \\ ${ }^{1}$ Department of Physics and Astronomy, Rice University, Houston, Texas 77005, USA \\ ${ }^{2}$ Rice Center for Quantum Materials, Rice University, Houston, Texas 77005, USA \\ ${ }^{3}$ Honeywell Quantum Solutions, 303 S. Technology Ct., Broomfield, Colorado 80021, USA
}

(Received 24 September 2020; revised 4 May 2021; accepted 27 July 2021; published 16 August 2021)

\begin{abstract}
Finite-size errors (FSEs), the discrepancies between an observable in a finite system and in the thermodynamic limit, are ubiquitous in numerical simulations of quantum many-body systems. Although a rough estimate of these errors can be obtained from a sequence of finite-size results, a strict, quantitative bound on the magnitude of FSE is still missing. Here we derive rigorous upper bounds on the FSE of local observables in real-time quantum dynamics simulations initialized from a product state. In $d$-dimensional locally interacting systems with a finite local Hilbert space, our bound implies $\left|\langle\hat{S}(t)\rangle_{L}-\langle\hat{S}(t)\rangle_{\infty}\right| \leqslant C(2 v t / L)^{c L-\mu}$, with $v, C, c, \mu$ constants independent of $L$ and $t$, which we compute explicitly. For periodic boundary conditions (PBCs), the constant $c$ is twice as large as that for open boundary conditions (OBCs), suggesting that PBCs have smaller FSEs than OBCs at early times. The bound can be generalized to a large class of correlated initial states as well. As a byproduct, we prove that the FSE of local observables in ground-state simulations decays exponentially with $L$ under a suitable spectral gap condition. Our bounds are practically useful in determining the validity of finite-size results, as we demonstrate in simulations of the one-dimensional (1D) quantum Ising and Fermi-Hubbard models.
\end{abstract}

DOI: 10.1103/PhysRevResearch.3.L032047

\section{INTRODUCTION}

Numerical simulations are crucial to our understanding of many-body quantum matter, and are routinely applied in all fields of physics and in chemistry. Unfortunately, many numerical techniques popular in these fields incur significant FSEs when approximating properties of a large (potentially infinite) system by properties of a finite one. The most direct example is exact diagonalization, which exactly solves the finite system numerically [1-4]. Accessible system sizes are limited since the Hilbert space dimension grows exponentially with system size; for the simplest case of interacting spin$1 / 2$ s, a state-of-the-art ground-state calculation is limited to $\sim 45$ spins [5]. FSEs also significantly affect other techniques, such as density matrix renormalization group (DMRG) [6-9], many tensor network algorithms [10,11], quantum dynamical typicality-based algorithms [12-16], and quantum Monte Carlo [17], and they are a significant source of errors for simulating quantum systems on quantum computers [18] and for analog quantum simulations using ultracold matter [19], trapped ions [20], and other platforms [21].

It is often difficult to characterize FSEs. The standard method to assess them is to calculate and compare observables for different system sizes, ideally using finite-size scaling [3].

\footnotetext{
*zhiyuan.wang@ @ice.edu

Published by the American Physical Society under the terms of the Creative Commons Attribution 4.0 International license. Further distribution of this work must maintain attribution to the author(s) and the published article's title, journal citation, and DOI.
}

Although useful, this method has limitations. One is that it offers no guarantees. Two different system sizes may have results that closely agree, but at larger sizes the physics changes and observables deviate [22]. Another is that one may not be able to study multiple system sizes that are sufficiently large to get a good estimate of the convergence.

In this paper, we derive rigorous upper bounds on the error of approximating observables in a large, possibly infinite, quantum many-body system by results in a smaller one. The bounds are applicable to arbitrary Hamiltonians for which a Lieb-Robinson (LR) bound exists. For quantum dynamics simulations starting from product initial states and evolving under locally interacting Hamiltonians with a finite local Hilbert space, the bound for a local observable $\hat{S}$ is

$$
\left|\langle\hat{S}(t)\rangle_{L}-\langle\hat{S}(t)\rangle_{\infty}\right| \leqslant C(2 v t / L)^{c L-\mu},
$$

where $v, C, c$, and $\mu$ are constants that can be computed explicitly and depend on the Hamiltonian, observable, and boundary condition.

Such dynamics is explored in a wide variety of ultracold matter experiments, such as quantum quenches and slow ramps in Rydberg atoms [23-28], molecules [29-31], Fermi gases [32], atoms in optical lattices [33-37], and optical clocks [38]. This dynamics can probe fundamental phenomena, such as many-body localization [39-43], prethermalization $[44,45]$, and generation of topological defects near critical points [46].

This bound is then extended to a large family of correlated initial states satisfying an exponential clustering condition. While our main focus is on dynamics, we also show that the FSE of local observables in a many-body ground state decays 


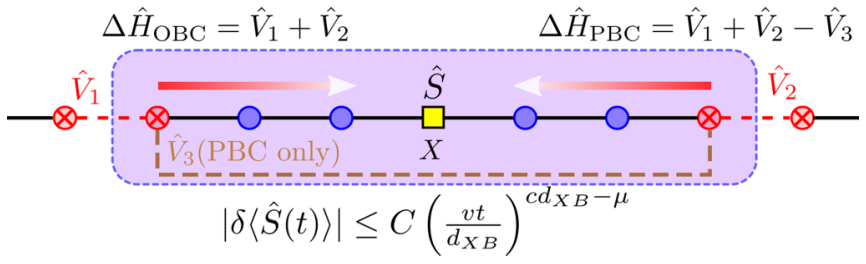

FIG. 1. An illustration of our bounds. In locally interacting systems, information propagates no faster than the LR speed $v$, so it takes a finite amount of time $t_{c} \sim d_{X B} / v$ for the effect of the boundary, $\Delta \hat{H}$ to affect the center site observable $\hat{S}$. Here $d_{X B}$ is the distance between the supports $X$ (yellow square) and $B$ (red crossed circles) of $\hat{S}(t)$ and $\Delta \hat{H}$, respectively.

exponentially in system size, under a suitable spectral gap condition.

The idea behind our bound is that locality-specifically, that one piece of a system does not instantly affect far-away pieces-imposes strong constraints on quantum dynamics $[47,48]$. This can be seen by considering evolution under a Hamiltonian initiated from a product state (other scenarios can be understood by similar arguments). As illustrated in Fig. 1, an observable in a region $X$ will be affected by FSEs only after a long enough time for information to propagate from the boundary to $X$. This idea is made precise by relating FSEs to unequal time correlation functions, which can then be bounded by a LR bound [49], a direct consequence of locality. Although similar ideas of applying LR bounds to analyze the performance of some numerical algorithms have been employed in Refs. [50-58], the connection to FSE has not been made explicit, and the practical utility of the bounds for numerics was not demonstrated. This idea has also been applied to estimate FSE in a nonrigorous way, for example, in Ref. [59].

Our FSE bound not only shows the convergence of finitesize approximations in principle, but is tight enough to be useful in practice, which we demonstrate in simulations of some prototypical models. For example, in the dynamics following a sudden change of parameters in a $1 D$ transverse field Ising model (TFIM) with $L=21$ sites, the error bounds for the transverse magnetization and nearest-neighbor correlations remain extremely small to times where they have evolved close to equilibrium. Furthermore, the bounds are reasonably tight: The time at which the error bound becomes significant is only $20-25 \%$ smaller than the time at which the actual FSE becomes noticeable. We similarly demonstrate this for the nonequilibrium relaxation of the Fermi-Hubbard model (FHM) from a checkerboard state, inspired by experiments and theory of Refs. [60-63]. The precision of these bounds is enabled by the major quantitative improvements offered by recent LR bounds [64,65].

In addition to their quantitative utility, these bounds provide insight into the convergence of numerical methods and open the way to designing new algorithms. One immediate consequence of the bounds is to rigorously show that the FSE decays exponentially with the linear dimension of the system for periodic boundary conditions (PBCs), as well as for open boundary conditions (OBCs) provided that one measures observables only near the center of the system, as commonly employed in the DMRG community. If one instead averages the measurement over all sites in OBCs, then our bound indicates that the error decays only algebraically. Similar behavior at finite temperature has been observed and analyzed in Ref. [66]. Furthermore, if one compares PBCs to OBCs with center site measurements, our error bound for PBCs decays twice as fast with distance as the bound for OBCs at early times, suggesting that $\mathrm{PBCs}$ give more reliable results at early times [67]. These insights may lead to new methods; one example is that they show why the moving-average cluster expansion method of Ref. [30] converges exponentially faster than alternative schemes.

\section{A. A simple bound for both OBCs and PBCs}

Consider the dynamical evolution of a quantum many-body system on an infinite $d$-dimensional lattice, governed by a locally interacting Hamiltonian $\hat{H}$. For illustrative purposes, in Fig. 1 we draw the configuration for a 1D nearest-neighbor interacting lattice model. Let $|\psi\rangle$ be the initial product state, $\hat{S}$ be a local observable to be measured that acts on a finite region $X$ (center point in Fig. 1), and let $\Delta \hat{H}=\sum_{j} \hat{V}_{j}$ be the sum of all the interaction terms between the inner and outer parts of the system (red links in Fig. 1). If PBCs are used, we further subtract from $\Delta \hat{H}$ the interaction between the first and the last site (brown link in Fig. 1). Let $\hat{H}_{L}$ and $\left|\psi_{L}\right\rangle$ denote the Hamiltonian and the initial state of the finite-size simulation, respectively (i.e., the restriction of $\hat{H}$ and $|\psi\rangle$ to the $L$-site inner system). Denote $\hat{H}^{\prime}=\hat{H}-\Delta \hat{H}$, so $\hat{H}^{\prime}$ decouples into two commuting terms, one acting only on the inner system, the other acting only on the outer system. The FSE of the observable $\hat{S}$ is

$$
\delta\langle\hat{S}(t)\rangle_{\psi} \equiv\left|\left\langle e^{i \hat{H}_{L} t} \hat{S} e^{-i \hat{H}_{L} t}\right\rangle_{\psi_{L}}-\left\langle e^{i \hat{H} t} \hat{S} e^{-i \hat{H} t}\right\rangle_{\psi}\right|,
$$

where $\langle A\rangle_{\psi} \equiv\langle\psi|A| \psi\rangle$, and we set $\hbar=1$ throughout. Since $\hat{H}^{\prime}$ decouples into two independent spatial regions (inner and outer) and $|\psi\rangle$ is a product state, the first term in Eq. (2) can be rewritten as $\left\langle e^{i \hat{H}^{\prime} t} \hat{S} e^{-i \hat{H}^{\prime} t}\right\rangle_{\psi}$. Inserted into Eq. (2), the two expectation values are taken in the same state $|\psi\rangle$, so their difference can be bounded by the operator norm $|\langle\psi|\hat{A}| \psi\rangle| \leqslant$ $\|\hat{A}\|$. Using the unitary invariance of operator norm $\|\hat{A}\|=$ $\|\hat{U} \hat{A} \hat{V}\|$ for arbitrary unitary operators $\hat{U}, \hat{V}$, we have

$$
\left|\delta\langle\hat{S}(t)\rangle_{\psi}\right| \leqslant\left\|\hat{U}_{I}(t) \hat{S} \hat{U}_{I}(t)^{\dagger}-\hat{S}\right\|,
$$

where $\hat{U}_{I}(t)=e^{-i \hat{H} t} e^{i \hat{H}^{\prime} t}$ is the evolution operator in the interaction picture, which satisfies $\hat{U}_{I}(0)=1$ and $i \partial_{t} \hat{U}_{I}(t)=$ $\hat{U}_{I}(t) \Delta \hat{H}(t)$, where $\Delta \hat{H}(t)=e^{-i \hat{H}^{\prime} t} \Delta \hat{H} e^{i \hat{H}^{\prime} t}$. Now applying the fundamental theorem of calculus and the triangle inequality, we obtain a bound on the FSE:

$$
\begin{aligned}
\left|\delta\langle\hat{S}(t)\rangle_{\psi}\right| & \leqslant \int_{0}^{t}\left\|\frac{d}{d t^{\prime}}\left[\hat{U}_{I}\left(t^{\prime}\right) \hat{S} \hat{U}_{I}\left(t^{\prime}\right)^{\dagger}-\hat{S}\right]\right\| d t^{\prime} \\
& =\int_{0}^{t}\left\|\hat{U}_{I}\left(t^{\prime}\right)\left[\Delta \hat{H}\left(t^{\prime}\right), \hat{S}\right] \hat{U}_{I}\left(t^{\prime}\right)^{\dagger}\right\| d t^{\prime} \\
& =\int_{0}^{t}\left\|\left[\Delta \hat{H}\left(t^{\prime}\right), \hat{S}\right]\right\| d t^{\prime} .
\end{aligned}
$$

The integrand is the quantity bounded by LR bounds, so to upper bound the FSE, one can insert the relevant LR 
bound. We focus on locally interacting systems, but Eq. (4) applies equally to long-range interactions by substituting the corresponding LR bounds [57,68-74] in those systems. For a locally interacting system, the currently tightest LR bound is obtained by computing the series in Eq. (S19) of the Supplemental Material (SM) [75], which is based on Refs. [64,65], although this may not be efficiently computable in general. A slightly looser but efficiently computable method is discussed in Ref. [65], in which one solves a system of first-order linear differential equations for a number of variables proportional to the system size. To see the qualitative features of the bound for large systems, we can insert the simple expression given in Eq. (3) of Ref. [65] into Eq. (4) to obtain

$$
\left|\delta\langle\hat{S}(t)\rangle_{\psi}\right| \leqslant \sum_{j} c_{j}\left(\frac{v|t|}{d_{X j}}\right)^{D\left(\hat{S}, \hat{V}_{j}\right)},
$$

where $c_{j}$ are constants independent of $t$ and $d_{X j}, D\left(\hat{S}, \hat{V}_{j}\right)$ is the distance between the operators $\hat{S}, \hat{V}_{j}$ in the commutativity graph (CG) as introduced in Ref. [65], and $v$ is the LR speed. The distance in the CG is related to the distance in real space $d_{X j}$ by $D\left(\hat{S}, \hat{V}_{j}\right)=\eta d_{X j}-\mu$, where $\eta, \mu$ are (straightforwardly determined) constants and $d_{X j}$ is the distance between $X$ and $j$ in real space. Therefore, the right-hand side of Eq. (5) is bounded by $\left(v|t| / d_{X B}\right)^{\eta d_{X B}-\mu}$, where $d_{X B}=$ $\min _{j \in B} d_{X j}$. Despite its simplicity, the $t$ dependence of this bound generically agrees with the exact error to lowest order in $t$ in OBCs [75].

Besides its practical utility for bounding FSEs in calculations, as demonstrated below, this result has qualitative implications. One is to rigorously support the common practice of measuring observables close to the center site in OBC numerics (e.g., in the DMRG community), rather than averaging over all sites. This minimizes the error bound, since the center size maximizes $d_{X B}$. This choice yields our main result in Eq. (1) for the OBC case, with $c=\eta / 2$. Our bound allows one to extend this. For example, in dimensions greater than one, we can minimize FSEs by choosing an optimal cluster shape that minimizes the right-hand side of Eq. (5) and run simulations on the optimal shape.

As a side note, although we have restricted our discussion to a local observable $\hat{S}$, our bounds can equally well be applied to nonlocal ones of finite extent, such as equal time correlation functions. To do this, one only needs to replace the local observable $\hat{S}$ in Eq. (4) by a nonlocal one, and measure $\hat{S}$ in the central region of the finite cluster so that $\hat{S}$ is as far from the boundary as possible. The resulting FSE would be bigger than local observables, but for 1D finite-size simulation with $\sim 20$ sites, one can simulate a $\sim 4$ site correlation function with acceptably small error bounds. Such correlations are important for many experiments.

\section{B. An improved bound for PBCs}

In the previous section, we treated $\mathrm{PBC}$ in a way similar to OBCs. But it turns out that the resulting bound in Eqs. (4) and (5) is qualitatively loose at small $t$ for PBCs. The reason for this can be intuitively understood as follows. The two terms on the right-hand side of Eq. (2) can be expanded in $t$. As we discuss in greater detail in the SM [75], the FSE for $\hat{S}(t)$ actually is only contributed by terms in $e^{i \hat{H} t} \hat{S} e^{-i \hat{H} t}$ whose spatial span is larger than $L$ and terms in $e^{i \hat{H}_{L} t} \hat{S}^{-i \hat{H}_{L} t}$ that wrap around the whole periodic system. The leading order of these terms is proportional to $t^{\mathcal{L}}$, where $\mathcal{L}$ is the length of the shortest noncontractible loop on the PBC CG, which is roughly twice as large as the exponent $D\left(\hat{S}, \hat{V}_{j}\right)$ in Eq. (5). The SM [75] extends methods developed in Refs. [64,65] to derive a rigorous upper bound for $|\delta\langle\hat{S}(t)\rangle|$ that leads to this improved $t^{\mathcal{L}}$ scaling. The main result is

$$
\left|\delta\langle\hat{S}(t)\rangle_{\psi}^{(\mathrm{PBC})}\right| \leqslant \sum_{1 \leqslant p \leqslant d} C_{p}\left(\frac{2 v_{p} t}{L_{p}}\right)^{\mathcal{L}_{p}},
$$

where the constant $C_{p}$ is given in Eq. (S52), $v_{p}$ is the LR speed in the $p$ th direction given in Eq. (S53), and $\mathcal{L}_{p}$ is the size of the periodic system in the $p$ th direction in CG. $\mathcal{L}_{p}$ is related to the real space system size $L_{p}$ by $\mathcal{L}_{p}=\eta_{p} L_{p}-\mu_{p}$ for constant integers $\eta_{p}, \mu_{p}$. We note that while this bound improves the small-time exponent of the PBC bound by a factor of 2 compared to Eq. (5), the timescale $t_{c} \approx \min _{p} L_{p} / 2 v_{p}$ on which the bound exponentially grows is still approximately the same as Eq. (5). Besides its quantitative utility, Eq. (6) shows that in anisotropic systems where $v_{p}$ is different in each direction, one should choose $L_{p} \propto v_{p}$ to minimize the FSE.

\section{FSEs in nondegenerate gapped ground states}

So far, we have been discussing FSEs of quantum dynamics simulations. We now derive a bound on FSEs of local observables in nondegenerate ground states under a gap assumption. This result is interesting in its own right, and will also be useful for our subsequent generalization of the dynamics error bound to correlated initial states.

The Hamiltonian $\hat{H}$, observable $\hat{S}$, boundary terms $\Delta \hat{H}$, etc. are the same as before. For convenience, we suppose that the operator $\hat{S}=\hat{S}_{l}$ has unit norm and acts nontrivially only within a region $X=X_{l}$ of diameter $l$ which sits on the center of the finite-size cluster. The difference now is that we consider the observable $\langle\hat{S}\rangle=\operatorname{Tr}[\hat{\rho} \hat{S}]$ given by the ground-state density matrix $\hat{\rho}$. The numerical simulation approximates this thermodynamic quantity by the expectation value in the finite-size ground state $\operatorname{Tr}\left[\hat{\rho}_{L} \hat{S}\right]$, where $\hat{\rho}_{L}$ is the ground-state density matrix of $\hat{H}_{L}$. Our result relies on an assumption that the interpolated Hamiltonain $\hat{H}(\lambda) \equiv \hat{H}-\lambda \Delta \hat{H}$ is nondegenerate for all $0 \leqslant \lambda \leqslant 1$ and has a uniform spectral gap $\min _{0 \leqslant \lambda \leqslant 1} \Delta(\lambda)=\Delta>0$. When this condition is satisfied, then analogously to Eq. (1) we have [75]

$$
\left|\operatorname{Tr}\left[\hat{\rho} \hat{S}_{l}-\hat{\rho}_{L} \hat{S}_{l}\right]\right| \leqslant C e^{-(L-l) / 2 \xi} .
$$

\section{Bounds for correlated initial states}

We now generalize our error bound to dynamics initiated from a class of (possibly mixed) initial states $\hat{\rho}$, for which there exists a sufficiently good finite size approximation $\hat{\rho}_{L}$ satisfying Eq. (7). This includes nondegenerate gapped ground states (under the condition described above) but also includes translation invariant matrix product states (MPSs) with a finite bond dimension [76] and finite-temperature thermal states [77] $\hat{\rho}=e^{-\beta \hat{H}} / \operatorname{Tr}\left[e^{-\beta \hat{H}}\right]$ above a certain tem- 
perature, where $\hat{\rho}_{L}=e^{-\beta \hat{H}_{L}} / \operatorname{Tr}\left[e^{-\beta \hat{H}_{L}}\right]$, i.e., the thermal state of $\hat{H}_{L}$.

Given that we have an initial state $\hat{\rho}_{L}$ satisfying Eq. (7), we can bound the dynamics FSE as

$$
\begin{aligned}
\delta\langle\hat{S}(t)\rangle_{\rho} & =\left|\left\langle\hat{S}_{L}(t)\right\rangle_{\rho_{L}}-\langle\hat{S}(t)\rangle_{\rho}\right| \\
& \leqslant\left|\operatorname{Tr}\left[\left(\hat{\rho}_{L}-\hat{\rho}_{[L]}\right) \hat{S}_{L}(t)\right]\right|+\left|\left\langle\hat{S}_{L}(t)-\hat{S}(t)\right\rangle_{\rho}\right|,
\end{aligned}
$$

where $\hat{S}_{L}(t)=e^{i \hat{H}_{L} t} \hat{S} e^{-i \hat{H}_{L} t}, \hat{\rho}_{[L]}$ is the reduced density matrix of $\hat{\rho}$ on the finite cluster, and in the second line we used the triangle inequality. The second term can be bounded using the same method as in Eq. (2), since $\operatorname{Tr}[\hat{\rho} \hat{A}] \leqslant\|\hat{A}\|$ for any density matrix $\hat{\rho}$. To bound the first term, we insert the expansion $\hat{S}_{L}(t)=\hat{S}_{0}(t)+\sum_{l=1}^{L}\left[\hat{S}_{l}(t)-\hat{S}_{l-1}(t)\right]$ into Eq. (8) and notice that $\hat{S}_{l}(t)-\hat{S}_{l-1}(t)$ is an operator acting on $X_{l}$, whose norm is bounded by Eqs. (4) and (5) to be $\left\|\hat{S}_{l}(t)-\hat{S}_{l-1}(t)\right\| \leqslant$ $C(2 v t / l)^{\eta l / 2}$ for some constant $C$. For initial states satisfying Eq. (7), this implies [75]

$$
\delta\langle\hat{S}(t)\rangle_{\rho} \leqslant C_{1} e^{(v t-L / 2) / \xi}+C_{2} e^{\eta(v t-L / 2)},
$$

where $C_{1}$ and $C_{2}$ are model-dependent constants that can be explicitly determined.

\section{E. Example: 1D TFIM}

We test our dynamics error bounds in simulations of prototypical models for quantum many-body physics, starting with the TFIM,

$$
\hat{H}=-J \sum_{j} \hat{\sigma}_{j}^{z} \hat{\sigma}_{j+1}^{z}-h \sum_{j} \hat{\sigma}_{j}^{x} .
$$

This is a canonical model for quantum phase transitions [78,79] and occurs in materials like $\mathrm{CoNb}_{2} \mathrm{O}_{6}$ [80], cold atom [26,46,81] and trapped ion [82-86] experiments, and superconducting circuits $[87,88]$. We numerically study the dynamics of this model at the critical point $J=h$ for several $L$, and calculate the exact evolution for $L=\infty$. Specifically, we study the dynamics of $\left\langle\hat{\sigma}^{x}(t)\right\rangle$ starting from $|\psi(0)\rangle=|\rightarrow \rightarrow \ldots \rightarrow\rangle$. Analogous dynamics in the 2D TFIM has been explored in Rydberg atom experiments [26,27].

Figure 2(a) shows $\left\langle\hat{\sigma}_{j}^{x}(t)\right\rangle$ from $L=5$ to 21 using PBCs, along with the exact $L=\infty$ solution [89]. To obtain a FSE bound for $\left\langle\hat{\sigma}_{j}^{x}(t)\right\rangle$, we use the LR bound given in Eq. (S24) of the SM [75] [obtained from the general bound Eq. (S20)], which, after inserting into Eq. (4), yields

$$
\left|\delta\left\langle\hat{\sigma}_{j}^{x}(t)\right\rangle\right| \leqslant 4 \sqrt{\frac{J}{h}} \frac{(2 \sqrt{J h} t)^{2 L-1}}{(2 L-1) !}+\frac{J}{h} \frac{(4 \sqrt{J h} t)^{2 L-2}}{(2 L-2) !} .
$$

As Fig. 2(a) shows, this error bound provides a guarantee of the numerical calculations' accuracy out to interesting and useful timescales. For the $L=21$-site calculation, the bound guarantees that the results are accurate (within $10^{-2}$ ) up to times $J t \sim 3.5$, where the observable has nearly reached equilibrium. Furthermore, this time is in reasonable accord with the true time at which FSE becomes important (within 20\%).

We emphasize that the FSE bound never made use of the TFIM's exact solution. The bound Eq. (4) can be applied to any system, including in dimensions greater than one. As we will now demonstrate in the 1D FHM, the bound still provides

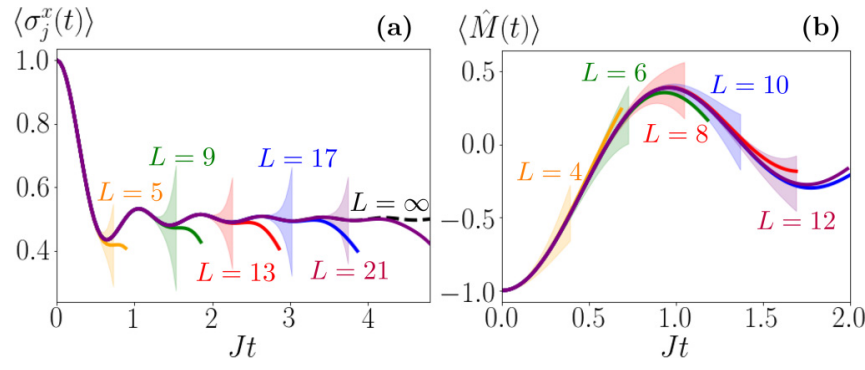

FIG. 2. Numerically exact evolution for (a) $\left\langle\hat{\sigma}_{j}^{x}(t)\right\rangle$ in the $L$-site PBC TFIM at $J=h$, with $|\psi(0)\rangle=|\rightarrow \rightarrow \ldots \rightarrow\rangle$, and (b) $\langle\hat{M}(t)\rangle$ in the $L$-site OBC FHM at $U=0.5 J$, with $|\psi(0)\rangle=|202020 \ldots 20\rangle$. The dashed curve in TFIM is the exact $L \rightarrow \infty$ result. The shaded areas for each curve represent the region in which the actual $L \rightarrow$ $\infty$ values must lie according to the FSE bounds in Eqs. (11), (4), and (S19), respectively, and we do not show the shade beyond the timescale $t_{c} \approx L /(2 v)$ at which the error bounds become too large to be useful.

a useful guarantee of the finite-size results when no exact solution is available.

\section{F. Example: FHM}

The 1D FHM describes spin- $1 / 2$ fermions in a lattice whose Hamiltonian is

$$
\hat{H}=-J \sum_{\langle i j\rangle, \sigma=\uparrow, \downarrow}\left(\hat{a}_{i \sigma}^{\dagger} \hat{a}_{j \sigma}+\text { H.c. }\right)+U \sum_{i} \hat{n}_{i}^{\uparrow} \hat{n}_{i}^{\downarrow} .
$$

The FHM exhibits rich behavior, such as a metal-Mott insulator transition, and potentially high-temperature superconductivity. It is a reasonable approximation of some real materials, such as $\mathrm{FeO}, \mathrm{NiO}, \mathrm{CoO}[90]$, and has been realized in ultracold atoms [91-95].

We numerically study the relaxation dynamics of a charge density wave state $|\psi(0)\rangle=|202020 \ldots 20\rangle$, analogous to previous theory [96] and experiments [97], where 2 (0) means a doubly occupied (empty) site. We run the finite-size simulations in OBCs and measure the density imbalance $\hat{M}(t)=$ $\left[\hat{N}_{\text {even }}(t)-\hat{N}_{\text {odd }}(t)\right] / L[96]$. To get a FSE bound for $\hat{M}(t)$, we use the currently tightest LR bound, obtained by numerically summing the series in Eq. (S19) of the SM [75] and inserting the result into Eq. (4). Figure 2(b) shows the results.

Our error bound can be compared to estimates of FSE obtained from comparing calculations of different sizes. For example, one can take the difference between the $L=10$ and $L=12$ as a rough estimate of the FSE of the $L=12$ calculation. Our bound is comparable in its guaranteed timescale of convergence to this conventional estimate. For example, we can guarantee that the FSE in $\langle\hat{M}(t)\rangle$ of the $L=12$ result is less than $1 \%$ for $J t \leqslant 1.2$, comparable to the time $J t \sim 1.6$ where the $L=10$ and 12 results differ noticeably.

\section{CONCLUSIONS}

We have presented a rigorous upper bound on the FSE of local observables measured in numerical simulations of quantum dynamics starting from a large class of initial states. 
For product initial states, the bounds show an advantage of using PBCs at early times. We also presented a generalization to simulations of local observables in nondegenerate gapped ground states. In all the cases we considered, the bounds decay exponentially in system size and guarantee the accuracy of finite-size dynamics simulation up to a timescale $t_{c} \sim L / 2 v$. These insights into FSE can motivate better algorithms.

The quantitative utility of the bounds is demonstrated in the 1D TFIM and 1D FHM. In both cases, the error bounds are extremely small up to timescales where there is interesting physics and even equilibration, and they are reasonably tight compared to the actual FSEs. We expect these bounds to provide useful tools to researchers going forward, providing FSE bounds on numerical calculations and suggesting new numerical methods that minimize this error.

\section{ACKNOWLEDGMENTS}

We thank Miles Stoudenmire, Miroslav Hopjan, Bhuvanesh Sundar and Ian White for discussions, and Brian Neyenhuis for a careful reading of the manuscript. This work was supported in part by the Welch Foundation (Grant No. C-1872), the National Science Foundation (Grant No. PHY-1848304), and the Office of Naval Research (Grant No. N00014-20-1-2695).
[1] N. Laflorencie and D. Poiblanc, Simulations of pure and doped low-dimensional spin-1/2 gapped systems, in Quantum Magnetism. Lecture Notes in Physics (Springer-Verlag, Berlin, Heidelberg, 2004).

[2] R. M. Noack and S. R. Manmana, Diagonalization- and numerical renormalization-group-based methods for interacting quantum systems, in Lectures on the Physics of Highly Correlated Electron Systems IX: Ninth Training Course in the Physics of Correlated Electron Systems and High-Tc Superconductors, edited by A. Avella and F. Mancini, AIP Conf. Proc. No. 789 (AIP, New York, 2005), p. 93.

[3] A. W. Sandvik, Computational studies of quantum spin systems, in Lectures on the Physics of Strongly Correlated Systems XIV: Fourteenth Training Course in the Physics of Strongly Correlated Systems, edited by A. Avella and F. Mancini, AIP Conf. Proc. No. 1297 (AIP, New York, 2010), p. 135.

[4] A. Läuchli, Numerical simulations of frustrated systems, in Introduction to Frustrated Magnetism: Materials, Experiments, Theory (Springer, Berlin, Heidelberg, 2011), pp. 481-511.

[5] Reaching even these system sizes is possible only if internal, translation, and point group symmetries are utilized, and if state-of-the-art algorithms and large-scale computational resources are employed. Researchers usually employ much smaller systems for computational convenience.

[6] S. R. White, Density Matrix Formulation for Quantum Renormalization Groups, Phys. Rev. Lett. 69, 2863 (1992).

[7] K. A. Hallberg, New trends in density matrix renormalization, Adv. Phys. 55, 477 (2006).

[8] U. Schollwöck, The density-matrix renormalization group in the age of matrix product states, Ann. Phys. 326, 96 (2011).

[9] E. M. Stoudenmire and S. R. White, Studying two-dimensional systems with the density matrix renormalization group, Annu. Rev. Condens. Matter Phys. 3, 111 (2012).

[10] D. Perez-Garcia, F. Verstraete, M. M. Wolf, and J. I. Cirac, Matrix product state representations, Quantum Inf. Comput. 7, 401 (2007).

[11] R. Orús, A practical introduction to tensor networks: Matrix product states and projected entangled pair states, Ann. Phys. 349, 117 (2014).

[12] C. Bartsch and J. Gemmer, Dynamical Typicality of Quantum Expectation Values, Phys. Rev. Lett. 102, 110403 (2009).
[13] T. A. Elsayed and B. V. Fine, Regression Relation for Pure Quantum States and Its Implications for Efficient Computing, Phys. Rev. Lett. 110, 070404 (2013).

[14] R. Steinigeweg, A. Khodja, H. Niemeyer, C. Gogolin, and J. Gemmer, Pushing the Limits of the Eigenstate Thermalization Hypothesis Towards Mesoscopic Quantum Systems, Phys. Rev. Lett. 112, 130403 (2014).

[15] R. Steinigeweg, J. Gemmer, and W. Brenig, Spin-Current Autocorrelations from Single Pure-State Propagation, Phys. Rev. Lett. 112, 120601 (2014).

[16] R. Steinigeweg, F. Heidrich-Meisner, J. Gemmer, K Michielsen, and H. De Raedt, Scaling of diffusion constants in the spin-12 XX ladder, Phys. Rev. B 90, 094417 (2014).

[17] Quantum Monte Carlo Methods in Physics and Chemistry, edited by M. P. Nightingale and C. J. Umrigar (Springer, Netherlands, 1999).

[18] A. M. Childs, D. Maslov, Y. Nam, N. J. Ross, and Y. Su, Toward the first quantum simulation with quantum speedup, Proc. Natl. Acad. Sci. 115, 9456 (2018).

[19] I. Bloch, J. Dalibard, and S. Nascimbène, Quantum simulations with ultracold quantum gases, Nat. Phys. 8, 267 (2012).

[20] R. Blatt and C. F. Roos, Quantum simulations with trapped ions, Nat. Phys. 8, 277 (2012).

[21] E. Altman, K. R. Brown, G. Carleo, L. D. Carr, E. Demler, C. Chin, B. DeMarco, S. E. Economou, M. A. Eriksson, KaiMei C. Fu, M. Greiner, K. R.A. Hazzard, R. G. Hulet, A. J. Kollár, B. L. Lev, M. D. Lukin, R. Ma, X. Mi, S. Misra, C. Monroe et al., Quantum simulators: Architectures and opportunities, PRX Quantum 2, 017003 (2021).

[22] J. Bausch, T. S. Cubitt, A. Lucia, D. Perez-Garcia, and M. M. Wolf, Size-driven quantum phase transitions, Proc. Natl. Acad. Sci. USA 115, 19 (2018).

[23] J. Zeiher, R. van Bijnen, P. Schauß, S. Hild, J.-Y. Choi, T. Pohl, I. Bloch, and C. Gross, Many-body interferometry of a Rydberg-dressed spin lattice, Nat. Phys. 12, 1095 (2016).

[24] N. Takei, C. Sommer, C. Genes, G. Pupillo, H. Goto, K. Koyasu, H. Chiba, M. Weidemüller, and K. Ohmori, Direct observation of ultrafast many-body electron dynamics in an ultracold Rydberg gas, Nat. Commun. 7, 13449 (2016). 
[25] H. Bernien, S. Schwartz, A. Keesling, H. Levine, A. Omran, H. Pichler, S. Choi, A. S. Zibrov, M. Endres, M. Greiner et al., Probing many-body dynamics on a 51-atom quantum simulator, Nature (London) 551, 579 (2017).

[26] E. Guardado-Sanchez, P. T. Brown, D. Mitra, T. Devakul, D. A. Huse, P. Schauß, and W. S. Bakr, Probing the Quench Dynamics of Antiferromagnetic Correlations in a 2D Quantum Ising Spin System, Phys. Rev. X 8, 021069 (2018).

[27] V. Lienhard, S. de Léséleuc, D. Barredo, T. Lahaye, A. Browaeys, M. Schuler, L.-P. Henry, and A. M. Läuchli, Observing the Space- and Time-Dependent Growth of Correlations in Dynamically Tuned Synthetic Ising Models with Antiferromagnetic Interactions, Phys. Rev. X 8, 021070 (2018).

[28] A. P. Orioli, A. Signoles, H. Wildhagen, G. Günter, J. Berges, S. Whitlock, and M. Weidemüller, Relaxation of an Isolated Dipolar-Interacting Rydberg Quantum Spin System, Phys. Rev. Lett. 120, 063601 (2018).

[29] B. Yan, S. A. Moses, B. Gadway, J. P. Covey, K. R. A. Hazzard, A. M. Rey, D. S. Jin, and J. Ye, Observation of dipolar spinexchange interactions with lattice-confined polar molecules, Nature (London) 501, 521 (2013).

[30] K. R. A. Hazzard, B. Gadway, M. Foss-Feig, B. Yan, S. A. Moses, J. P. Covey, N. Y. Yao, M. D. Lukin, J. Ye, D. S. Jin, and A. M. Rey, Many-Body Dynamics of Dipolar Molecules in an Optical Lattice, Phys. Rev. Lett. 113, 195302 (2014).

[31] F. Seeßelberg, X.-Y. Luo, M. Li, R. Bause, S. Kotochigova, I. Bloch, and C. Gohle, Extending Rotational Coherence of Interacting Polar Molecules in a Spin-Decoupled Magic Trap, Phys. Rev. Lett. 121, 253401 (2018).

[32] S. Smale, P. He, B. A. Olsen, K. G. Jackson, H. Sharum, S. Trotzky, J. Marino, A. M. Rey, and J. H. Thywissen, Observation of a transition between dynamical phases in a quantum degenerate Fermi gas, Sci. Adv. 5, eaax1568 (2019).

[33] A. de Paz, A. Sharma, A. Chotia, E. Marechal, J. H. Huckans, P. Pedri, L. Santos, O. Gorceix, L. Vernac, and B. Laburthe-Tolra, Nonequilibrium Quantum Magnetism in a Dipolar Lattice Gas, Phys. Rev. Lett. 111, 185305 (2013).

[34] C. Meldgin, U. Ray, P. Russ, D. Chen, D. M. Ceperley, and B. DeMarco, Probing the Bose glass-superfluid transition using quantum quenches of disorder, Nat. Phys. 12, 646 (2016).

[35] J.-Y. Choi, S. Hild, J. Zeiher, P. Schauß, A. Rubio-Abadal, T. Yefsah, V. Khemani, D. A. Huse, I. Bloch, and C. Gross, Exploring the many-body localization transition in two dimensions, Science 352, 1547 (2016).

[36] P. Bordia, H. Lüschen, S. Scherg, S. Gopalakrishnan, M. Knap, U. Schneider, and I. Bloch, Probing Slow Relaxation and ManyBody Localization in Two-Dimensional Quasiperiodic Systems, Phys. Rev. X 7, 041047 (2017).

[37] L. Gabardos, B. Zhu, S. Lepoutre, A. M. Rey, B. LaburtheTolra, and L. Vernac, Relaxation of the Collective Magnetization of a Dense 3D Array of Interacting Dipolar $s=3$ Atoms, Phys. Rev. Lett. 125, 143401 (2020).

[38] A. Goban, R. B. Hutson, G. E. Marti, S. L. Campbell, M. A. Perlin, P. S. Julienne, J. P. D'Incao, A. M. Rey, and J. Ye, Emergence of multi-body interactions in a fermionic lattice clock, Nature (London) 563, 369 (2018).

[39] J. Eisert, M. Friesdorf, and C. Gogolin, Quantum many-body systems out of equilibrium, Nat. Phys. 11, 124 (2015).
[40] R. Nandkishore and D. A. Huse, Many-body localization and thermalization in quantum statistical mechanics, Annu. Rev. Condens. Matter Phys. 6, 15 (2015).

[41] D. J. Luitz, N. Laflorencie, and F. Alet, Extended slow dynamical regime close to the many-body localization transition, Phys. Rev. B 93, 060201(R) (2016).

[42] D. J. Luitz and Y. B. Lev, The ergodic side of the many-body localization transition, Ann. Phys. (Berlin) 529, 1600350 (2017).

[43] S. A. Parameswaran and R. Vasseur, Many-body localization, symmetry and topology, Rep. Prog. Phys. 81, 082501 (2018).

[44] T. Mori, T. N. Ikeda, E. Kaminishi, and M. Ueda, Thermalization and prethermalization in isolated quantum systems: A theoretical overview, J. Phys. B 51, 112001 (2018).

[45] C.-M. Schmied, A. N. Mikheev, and T. Gasenzer, Non-thermal fixed points: Universal dynamics far from equilibrium, Int. J. Mod. Phys. A 34, 1941006 (2019).

[46] J. Simon, W. S. Bakr, R. Ma, M. E. Tai, P. M. Preiss, and M. Greiner, Quantum simulation of antiferromagnetic spin chains in an optical lattice, Nature (London) 472, 307 (2011).

[47] S. Bravyi, M. B. Hastings, and F. Verstraete, Lieb-Robinson Bounds and the Generation of Correlations and Topological Quantum Order, Phys. Rev. Lett. 97, 050401 (2006).

[48] M. B. Hastings, Locality in quantum systems, arXiv:1008.5137.

[49] E. H. Lieb and D. W. Robinson, The finite group velocity of quantum spin systems, Commun. Math. Phys. 28, 251 (1972).

[50] T. J. Osborne, Efficient Approximation of the Dynamics of One-Dimensional Quantum Spin Systems, Phys. Rev. Lett. 97, 157202 (2006).

[51] T. J. Osborne, Simulating adiabatic evolution of gapped spin systems, Phys. Rev. A 75, 032321 (2007).

[52] T. J. Osborne, Ground state of a class of noncritical onedimensional quantum spin systems can be approximated efficiently, Phys. Rev. A 75, 042306 (2007).

[53] M. Kliesch, C. Gogolin, and J. Eisert, Lieb-Robinson bounds and the simulation of time-evolution of local observables in lattice systems, in Many-Electron Approaches in Physics, Chemistry and Mathematics (Springer International Publishing, Switzerland, 2014), pp. 301-318.

[54] M. P. Woods, M. Cramer, and M. B. Plenio, Simulating Bosonic Baths with Error Bars, Phys. Rev. Lett. 115, 130401 (2015).

[55] M. P. Woods and M. B. Plenio, Dynamical error bounds for continuum discretisation via Gauss quadrature rules-a LiebRobinson bound approach, J. Math. Phys. 57, 022105 (2016).

[56] J. Haah, M. Hastings, R. Kothari, and G. H. Low, Quantum algorithm for simulating real time evolution of lattice Hamiltonians, in 2018 IEEE 59th Annual Symposium on Foundations of Computer Science (FOCS) (IEEE, New York, 2018), pp. 350-360.

[57] M. C. Tran, A. Y. Guo, Y. Su, J. R. Garrison, Z. Eldredge, M. Foss-Feig, A. M. Childs, and A. V. Gorshkov, Locality and Digital Quantum Simulation of Power-Law Interactions, Phys. Rev. X 9, 031006 (2019).

[58] Y. Huang, Computing local properties in the trivial phase, arXiv:2001.10763.

[59] C. B. Dağ and K. Sun, Dynamical crossover in the transient quench dynamics of short-range transverse-field Ising models, Phys. Rev. B 103, 214402 (2021).

[60] M. Cramer, A. Flesch, I. P. McCulloch, U. Schollwöck, and J. Eisert, Exploring Local Quantum Many-Body Relaxation by 
Atoms in Optical Superlattices, Phys. Rev. Lett. 101, 063001 (2008).

[61] T. Enss and J. Sirker, Light cone renormalization and quantum quenches in one-dimensional Hubbard models, New J. Phys. 14, 023008 (2012).

[62] S. Trotzky, Y.-A. Chen, A. Flesch, I. P. McCulloch, U. Schollwöck, J. Eisert, and I. Bloch, Probing the relaxation towards equilibrium in an isolated strongly correlated onedimensional bose gas, Nat. Phys. 8, 325 (2012).

[63] A. Bauer, F. Dorfner, and F. Heidrich-Meisner, Temporal decay of Néel order in the one-dimensional Fermi-Hubbard model, Phys. Rev. A 91, 053628 (2015).

[64] C.-F. Chen and A. Lucas, Operator growth bounds from graph theory, Commun. Math. Phys. 385, 1273 (2021).

[65] Z. Wang and K. R. A. Hazzard, Tightening the Lieb-Robinson bound in locally interacting systems, PRX Quantum 1, 010303 (2020).

[66] D. Iyer, M. Srednicki, and M. Rigol, Optimization of finite-size errors in finite-temperature calculations of unordered phases, Phys. Rev. E 91, 062142 (2015).

[67] While the fact that the error bound for PBCs is smaller than that for OBCs does not necessarily imply that the actual FSE in PBC is smaller, in the Supplemental Material [75] we use short-time perturbative arguments to show that the actual error in PBC is indeed smaller at early times for most initial product states.

[68] M. B. Hastings and T. Koma, Spectral gap and exponential decay of correlations, Commun. Math. Phys. 265, 781 (2006).

[69] P. Richerme, Z.-X. Gong, A. Lee, C. Senko, J. Smith, M. FossFeig, S. Michalakis, A. V. Gorshkov, and C. Monroe, Non-local propagation of correlations in quantum systems with long-range interactions, Nature (London) 511, 198 (2014).

[70] Z.-X. Gong, M. Foss-Feig, S. Michalakis, and A. V. Gorshkov, Persistence of Locality in Systems with Power-Law Interactions, Phys. Rev. Lett. 113, 030602 (2014).

[71] M. Foss-Feig, Z.-X. Gong, C. W. Clark, and A. V. Gorshkov, Nearly Linear Light Cones in Long-Range Interacting Quantum Systems, Phys. Rev. Lett. 114, 157201 (2015).

[72] C.-F. Chen and A. Lucas, Finite Speed of Quantum Scrambling with Long Range Interactions, Phys. Rev. Lett. 123, 250605 (2019).

[73] T. Kuwahara and K. Saito, Strictly Linear Light Cones in LongRange Interacting Systems of Arbitrary Dimensions, Phys. Rev. X 10, 031010 (2020).

[74] M. C. Tran, C.-F. Chen, A. Ehrenberg, A. Y. Guo, A. Deshpande, Y. Hong, Z.-X. Gong, A. V. Gorshkov, and A. Lucas, Hierarchy of Linear Light Cones with Long-Range Interactions, Phys. Rev. X 10, 031009 (2020).

[75] See Supplemental Material at https://link.aps.org/supplemental/ 10.1103/PhysRevResearch.3.L032047 for the comparison of the error bounds to perturbation theory at early times, the derivation of the PBC error bound in Eq. (6), and the detailed proof of Eqs. (7) and (9). The PBC error bound Eq. (6) is derived from an important intermediate result Eq. (S18), for which we introduce three different methods to bound its righthand side. The numerically tightest one is given in Eq. (S20), a more efficiently computable one is given in Eq. (S39), and the constants for the simplest one Eq. (6) are given in Eqs. (S52), (S53), and (S55). The PBC error bound for TFIM in Eq. (11) is derived in Eqs. (S22-S25), and the OBC error bound for FHM is obtained by numerically summing the series in Eq. (S19) on the CG shown in Figure S2.

[76] For translation invariant MPS with a finite bond dimension, $\hat{\rho}_{L}$ can be taken as the $L$-site periodic version of $\hat{\rho}$. That $\hat{\rho}_{L}$ satisfies the condition in Eq. (7) can be proved using the transfer operator method which is used to prove that MPS has finite correlation length, see, e.g., Refs. [[8,10,11]]. The parameter $2 \xi$ can simply be taken as the correlation length of the MPS.

[77] M. Kliesch, C. Gogolin, M. J. Kastoryano, A. Riera, and J. Eisert, Locality of Temperature, Phys. Rev. X 4, 031019 (2014).

[78] M. Vojta, Quantum phase transitions, Rep. Prog. Phys. 66, 2069 (2003).

[79] S. Sachdev, Quantum Phase Transitions, 2nd ed. (Cambridge University Press, New York, 2011).

[80] R. Coldea, D. A. Tennant, E. M. Wheeler, E. Wawrzynska, D. Prabhakaran, M. Telling, K. Habicht, P. Smeibidl, and K. Kiefer, Quantum criticality in an Ising chain: Experimental evidence for emergent E8 symmetry, Science 327, 177 (2010).

[81] H. Labuhn, D. Barredo, S. Ravets, S. De Léséleuc, T. Macrì, T. Lahaye, and A. Browaeys, Tunable two-dimensional arrays of single Rydberg atoms for realizing quantum Ising models, Nature (London) 534, 667 (2016).

[82] A. Friedenauer, H. Schmitz, J. T. Glueckert, D. Porras, and T. Schätz, Simulating a quantum magnet with trapped ions, Nat. Phys. 4, 757 (2008).

[83] K. Kim, M.-S. Chang, S. Korenblit, R. Islam, E. E. Edwards, J. K. Freericks, G.-D. Lin, L.-M. Duan, and C. Monroe, Quantum simulation of frustrated Ising spins with trapped ions, Nature (London) 465, 590 (2010).

[84] K. Kim, S. Korenblit, R. Islam, E. E. Edwards, M. S. Chang, C. Noh, H. Carmichael, G. D. Lin, L. M. Duan, C. C. J. Wang et al., Quantum simulation of the transverse Ising model with trapped ions, New J. Phys. 13, 105003 (2011).

[85] B. P. Lanyon, C. Hempel, D. Nigg, M. Müller, R. Gerritsma, F. Zähringer, P. Schindler, J. T. Barreiro, M. Rambach, G. Kirchmair et al., Universal digital quantum simulation with trapped ions, Science 334, 57 (2011).

[86] J. W. Britton, B. C. Sawyer, A. C. Keith, C.-C. J. Wang, J. K. Freericks, H. Uys, M. J. Biercuk, and J. J. Bollinger, Engineered two-dimensional Ising interactions in a trapped-ion quantum simulator with hundreds of spins, Nature (London) 484, 489 (2012).

[87] R. Barends, A. Shabani, L. Lamata, J. Kelly, A. Mezzacapo, U. Las Heras, R. Babbush, A. G. Fowler, B. Campbell, Yu Chen et al., Digitized adiabatic quantum computing with a superconducting circuit, Nature (London) 534, 222 (2016).

[88] R. Harris, Y. Sato, A. J. Berkley, M. Reis, F. Altomare, M. H. Amin, K. Boothby, P. Bunyk, C. Deng, C. Enderud et al., Phase transitions in a programmable quantum spin glass simulator, Science 361, 162 (2018).

[89] J. Dziarmaga, Dynamics of a Quantum Phase Transition: Exact Solution of the Quantum Ising Model, Phys. Rev. Lett. 95, 245701 (2005).

[90] V. I. Anisimov, J. Zaanen, and O. K. Andersen, Band theory and Mott insulators: Hubbard $U$ instead of Stoner I, Phys. Rev. B 44, 943 (1991).

[91] T. Esslinger, Fermi-Hubbard physics with atoms in an optical lattice, Annu. Rev. Condens. Matter Phys. 1, 129 (2010).

[92] M. F. Parsons, A. Mazurenko, C. S. Chiu, G. Ji, D. Greif, and M. Greiner, Site-resolved measurement of the spin-correlation 
function in the Fermi-Hubbard model, Science 353, 1253 (2016).

[93] M. Boll, T. A. Hilker, G. Salomon, A. Omran, J. Nespolo, L. Pollet, I. Bloch, and C. Gross, Spin-and density-resolved microscopy of antiferromagnetic correlations in Fermi-Hubbard chains, Science 353, 1257 (2016).

[94] A. Mazurenko, C. S. Chiu, G. Ji, M. F. Parsons, M. KanászNagy, R. Schmidt, F. Grusdt, E. Demler, D. Greif, and M. Greiner, A cold-atom Fermi-Hubbard antiferromagnet, Nature (London) 545, 462 (2017).

[95] P. T. Brown, D. Mitra, E. Guardado-Sanchez, R. Nourafkan, A. Reymbaut, C.-D. Hébert, S. Bergeron, A.-M. S. Tremblay,
J. Kokalj, D. A. Huse et al., Bad metallic transport in a cold atom Fermi-Hubbard system, Science 363, 379 (2019).

[96] N. Schlünzen, J.-P. Joost, F. Heidrich-Meisner, and M. Bonitz, Nonequilibrium dynamics in the one-dimensional FermiHubbard model: Comparison of the nonequilibrium Greenfunctions approach and the density matrix renormalization group method, Phys. Rev. B 95, 165139 (2017).

[97] D. Pertot, A. Sheikhan, E. Cocchi, L. A. Miller, J. E. Bohn, M. Koschorreck, M. Köhl, and C. Kollath, Relaxation Dynamics of a Fermi Gas in an Optical Superlattice, Phys. Rev. Lett. 113, 170403 (2014). 